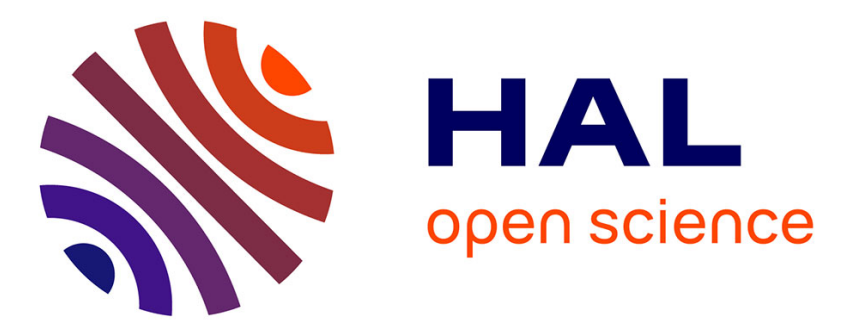

\title{
Monte-Carlo and Domain-Deformation Sensitivities
}

Paule Lapeyre, Stéphane Blanco, Cyril Caliot, Jérémi Dauchet, Mouna

El-Hafi, Richard A Fournier, Olivier Farges, Maxime Roger

\section{To cite this version:}

Paule Lapeyre, Stéphane Blanco, Cyril Caliot, Jérémi Dauchet, Mouna El-Hafi, et al.. Monte-Carlo and Domain-Deformation Sensitivities. RAD-19 - 9th International Symposium on Radiative Transfer, Jun 2019, Athens, Greece. 8 p. hal-02265080

\section{HAL Id: hal-02265080 https://imt-mines-albi.hal.science/hal-02265080}

Submitted on 12 Sep 2019

HAL is a multi-disciplinary open access archive for the deposit and dissemination of scientific research documents, whether they are published or not. The documents may come from teaching and research institutions in France or abroad, or from public or private research centers.
L'archive ouverte pluridisciplinaire HAL, est destinée au dépôt et à la diffusion de documents scientifiques de niveau recherche, publiés ou non, émanant des établissements d'enseignement et de recherche français ou étrangers, des laboratoires publics ou privés. 


\title{
MONTE-CARLO AND DOMAIN-DEFORMATION SENSITIVITIES
}

\author{
P. Lapeyre,,${ }^{1, *}$ S. Blanco, ${ }^{2}$ C. Caliot, ${ }^{1}$ J. Dauchet,${ }^{3}$ M. El Hafi, ${ }^{4}$ R. Fournier, ${ }^{2}$ O. Farges, ${ }^{5}$ M. Roger $^{6}$ \\ ${ }^{1}$ PROMES CNRS, Université Perpignan Via Domitia - 7, rue du Four Solaire, 66120 Font Romeu \\ Odeillo, France \\ ${ }^{2}$ LAPLACE, UMR 5213 - Université Paul Sabatier, 118, Route de Narbonne - 31062 Toulouse \\ Cedex, France \\ ${ }^{3}$ Université Clermont Auvergne, CNRS, SIGMA Clermont, Institut Pascal, F-63000 \\ Clermont-Ferrand, France \\ ${ }^{4}$ Université Fédérale de Toulouse Midi-Pyrénées, Mines Albi, UMR CNRS 5302, Centre \\ RAPSODEE, Campus Jarlard, F-81013 Albi CT Cedex \\ ${ }^{5}$ LEMTA - UMR 7563 - Université de Lorraine, Vandœuvre-lès-Nancy, France \\ 6 Univ Lyon, CNRS, INSA-Lyon, Université Claude Bernard Lyon 1, CETHIL UMR5008, F-69621, \\ Villeurbanne, France
}

\begin{abstract}
We address the question of evaluating shape derivatives of objective functions for radiative-transfer engineering involving semi-transparent media. After recalling the standard MonteCarlo approach to sensitivity estimation and its current limitations, a new method is presented for the specific case of geometrical sensitivities. This method is then tested in a square cavity filled by a multiple-scattering and absorbing (non-emitting) semi-transparent medium, irradiated by an emissive cylinder. A new geometrical sensitivity algorithm is presented with full genericity in order to allow its future implementation in complex geometries.
\end{abstract}

\section{INTRODUCTION}

The optimization of engineering processes involves an objective function driven by physical mechanisms. In the field of geometrical design, shape optimization models are required for one reason: to reach an extremum of a formulated objective function, stated here as $J(\vec{\pi})$, where $\vec{\pi}$ represents the design parameters vector. Radiative transfer involved in solar processes has a major influence on heat transfer models, and therefore on the objective function. Monte-Carlo methods are preferred for complex geometry process simulations where radiative transfer is preponderant [1]. Optimization methods, such as the gradient descent method, can provide information on a local extremum, and stochastic methods (genetic algorithms, particle swarm optimization) can inform on a global extremum [2-4]. In any case, the derivative of $J(\vec{\pi})$ with respect to $\vec{\pi}$ is a valuable piece of information for the optimization of engineering processes. In this paper, the Monte-Carlo method is used because of its ability to estimate physical quantities and their derivatives formulated as multiple integrals. We shall focus here on one selected design parameter $\pi$, selected in relation to the geometry. It has been shown in [5] that when a function is stated in an integral form $[1,6]$, the use of the Monte-Carlo method to calculate its sensitivity to geometrical parameters often leads to formalization and implementation difficulties. In this paper a new method to estimate geometrical sensitivity is presented which allows previously unsolvable configurations to be treated. This method

${ }^{*}$ Corresponding P. Lapeyre: paule.lapeyre@ promes.cnrs.fr 
focuses on the formulation of a specific model for the sensitivity [7], with the proper boundary conditions. First of all, the Monte-Carlo method used to estimate an objective function $J(\pi)$ and its derivative $\partial_{\pi} J(\pi)$ will be briefly reviewed. Then the new sensitivity model will be presented and its application on an academic configuration will be implemented to estimate local absorbed power $J(\pi)$ at any location within a multiple-scattering (non-emitting) semi-transparent medium and its derivative with respect to geometric parameter $\pi$.

\subsection{Estimating sensitivities inside an existing Monte-Carlo algorithm}

Any quantity $J$ expressed in its integral form can be estimated by a Monte-Carlo method as long as a random variable $\Gamma$, established over a multiple dimension domain $\mathcal{D}_{\Gamma}$ according to a probability density function $p_{\Gamma}(\gamma)$, is identified.

$$
J(\pi)=\int_{\mathcal{D}_{\Gamma}(\pi)} p_{\Gamma}(\gamma, \pi) d \gamma \hat{w}(\gamma, \pi)=\mathbb{E}(\hat{w}(\Gamma, \pi))
$$

$J$ is formulated as an expectation of the function $\hat{w}$ of the random variable $\Gamma$ which represents an opticalpath. To fully explicit the sampling of the probability density function $p_{\Gamma}(\gamma, \pi)$ we should develop the integral regarding to the number of events that form the optical path. The interested reader should find relevant details in [1] and [8]. The integration domain $\mathcal{D}_{\Gamma}(\pi)$ is the space of all possible optical-paths which depends strongly on the considered geometry. Depending on the context the dimension of $\mathcal{D}_{\Gamma}(\pi)$ can be high, for example it can be infinite in the presence of a scattering medium. The expectation is estimated numerically by the average value of the random variable function realizations $\hat{w}\left(\gamma_{i}\right)$ with a large number $N$ of realizations $\gamma_{i}$ from $\Gamma$ [9]. $J$ is an integral radiative quantity that may depend on the parameter $\pi$ through the integration domain, probability density function or the Monte-Carlo weight (1).

When the integration domain is independent of $\pi$ it is shown in [10] that the sensitivity of $J(\pi)$ with respect to $\pi$ is :

$$
\partial_{\pi} J(\pi)=\int_{\mathcal{D}_{\Gamma}} p_{\Gamma}(\gamma, \pi) d \gamma\left[\frac{\partial_{\pi} p_{\Gamma}(\gamma, \pi)}{p_{\Gamma}(\gamma, \pi)} \hat{w}(\gamma, \pi)+\partial_{\pi} \hat{w}(\gamma, \pi)\right]
$$

$\partial_{\pi} J(\pi)$ is an expectation of the function $\hat{w}_{\pi}$ of the random variable $\Gamma$.

$$
\begin{gathered}
\partial_{\pi} J(\pi)=\mathbb{E}\left(\hat{w}_{\pi}(\Gamma, \pi)\right) \\
\hat{w}_{\pi}(\gamma, \pi)=\left[\frac{\partial_{\pi} p_{\Gamma}(\gamma, \pi)}{p_{\Gamma}(\gamma, \pi)} \hat{w}(\gamma, \pi)+\partial_{\pi} \hat{w}(\gamma, \pi)\right]
\end{gathered}
$$

In this case both $J(\pi)$ and $\partial_{\pi} J(\pi)$ are expectations defined by the same random variable $\Gamma$ over the same domain $\mathcal{D}_{\Gamma}$ and sampled according to the same probability density function $p_{\Gamma}(\gamma, \pi)$. Only the Monte-Carlo weight functions are different. Numerically both expectations of radiative quantity and its derivatives are estimated by the same set of samples $\gamma_{i}$ from $p_{\Gamma}(\gamma, \pi)$. This formulation leads to the same algorithm for simultaneously computing $J(\pi)$ and $\partial_{\pi} J(\pi)$. The additional computation time is relatively small when compared to the evaluation of $J(\pi)$ alone $[10,11]$.

\subsection{The specific case of geometrical sensitivities}

When $\pi$ configures the geometry of the problem, the integration domain is a function of $\pi: \mathcal{D}_{\Gamma}(\pi)$ [12]. Following the sensitivities calculation using the Monte-Carlo method presented in [10] (Eq. (2)), the methodology is then extended to sensitivities with parameter $\pi$ defining the integration domain in $[12,13]$ :

$$
\partial_{\pi} J(\pi)=\int_{\mathcal{D}_{\Gamma}(\pi)} p_{\Gamma}(\gamma, \pi) d \gamma\left[\partial_{\pi} \hat{w}(\gamma, \pi)+\hat{w}(\gamma, \pi) \frac{\partial_{\pi} p_{\Gamma}(\gamma, \pi)}{p_{\Gamma}(\gamma, \pi)}+\frac{\vec{\nabla}\left(\hat{w}(\gamma, \pi) p_{\Gamma}(\gamma, \pi) \vec{v}_{\pi}\right)}{p_{\Gamma}(\gamma, \pi)}\right]
$$


The divergence term in the Monte-Carlo weight is a consequence of integration-domain differentiation using the Green-Ostrogradski theorem [11]. The deformation velocity vector $\vec{v}_{\pi}$ and $\vec{\nabla}$ operator have the same dimensions as $\mathcal{D}_{\Gamma}(\pi)$. The deformation velocity vector is formulated in [12] for academic configurations, e.g. to estimate the radiative absorption sensitivity of a slab with respect to its thickness. In [13] and [5] the difficulties of the method are highlighted and classified. Two major problems are pointed out. The first one lies in the difficulty of the deformation velocity vector terms and divergent terms construction once the integral formulation of the sensitivity (5) does not clearly appear. The second one concern the implementation difficulties that arise when it is applied on scattering media. Given these constraints the configuration used in this study accepts the fact that the deformation-velocity method can not be applied. The following section focuses on a new proposal. This time the sensitivities are obtained through differentiation of the radiative transfer model itself.

\section{RESTARTING FROM THE SENSITIVITY MODEL}

In the following section, instead of derivating the integral expectation formulation of $J(\pi)$ to estimate geometric sensitivities, we construct a sensitivity model by derivating the radiative transfer equation. Indeed, the quantity $\partial_{\pi} I$ leads directly to $\partial_{\pi} J$ when it is stated that $\partial_{\pi} J$ depends linearly on $\partial_{\pi} I^{1}$. Thus the main focus of the work is to construct a model of $\partial_{\pi} I$ with the proper boundary conditions. In this work the radiative transfer equation (RTE) in a scattering, absorbing and non-emissive medium with a boundary condition stated in intensity is written :

$$
\left\{\begin{array}{l}
\vec{u} \cdot \vec{\nabla} I(\vec{x}, \vec{u}, \pi)=-\left(k_{a}+k_{s}\right) I(\vec{x}, \vec{u}, \pi)+k_{s} \int_{4 \pi} p\left(\vec{u}^{\prime} \mid \vec{u}\right) d \vec{u}^{\prime} I\left(\vec{x}, \vec{u}^{\prime}, \pi\right) \text { for } \vec{x} \in \Omega \\
I(\vec{x}, \vec{u}, \pi)=I_{\partial \Omega(\pi)}(\vec{x}, \vec{u}, \pi) \text { for } \vec{x} \in \partial \Omega(\pi) \text { and } \vec{u} \cdot \vec{n}>0
\end{array}\right.
$$

The integral formulation of the solution $I(\vec{x}, \vec{u})$ of equation $(6)$ is formulated as an expectation of the random variable function $\hat{w}\left(I_{\partial \Omega(\pi)}\left(\vec{x}_{\Gamma}, \vec{u}_{\Gamma}\right)\right)$ of the radiative boundary condition.

$$
I(\vec{x}, \vec{u})=\int_{\mathcal{D}_{\Gamma}(\pi)} p_{\Gamma}\left(\vec{x}_{\gamma}, \vec{u}_{\gamma} \mid \vec{x}, \vec{u}\right) d \gamma \hat{w}\left(I_{\partial \Omega(\pi)}\left(\vec{x}_{\gamma}, \vec{u}_{\gamma}\right)\right)
$$

The RTE differentiation is stated as Eq. (8). Since $\pi$ only appears in the boundary conditions it does not affect the operators in Eq. (8) :

$$
\left\{\begin{array}{l}
\vec{u} \cdot \vec{\nabla} s(\vec{x}, \vec{u}, \pi)=-\left(k_{a}+k_{s}\right) s(\vec{x}, \vec{u}, \pi)+k_{s} \int_{4 \pi} p(\vec{u} \mid \vec{u}) d \vec{u}^{\prime} s\left(\vec{x}, \vec{u}^{\prime}, \pi\right) \\
s(\vec{x}, \vec{u}, \pi)=s_{\partial \Omega(\pi)}(\vec{x}, \vec{u}, \pi) \text { for } \vec{x} \in \partial \Omega(\pi) \text { and } \vec{u} \cdot \vec{n}>0
\end{array}\right.
$$

where $s(\vec{x}, \vec{u}, \pi)=\partial_{\pi} I(\vec{x}, \vec{u}, \pi)$. Equation (8) is identical to Eq. (6), since the sensitivity is transported by the same transport phenomena as the intensity. Consequently the integral solution of the sensitivity transport equation is stated as an expectation of a random variable function $s_{\partial \Omega(\pi)}\left(\vec{x}_{\Gamma_{\pi}}, \vec{u}_{\Gamma_{\pi}}\right)$ of the sensitivity boundary condition:

$$
s(\vec{x}, \vec{u}, \pi)=\int_{\mathcal{D}_{\Gamma_{\pi}}} p_{\Gamma_{\pi}}\left(\vec{x}_{\gamma_{\pi}}, \vec{u}_{\gamma_{\pi}} \mid \vec{x}, \vec{u}\right) d \gamma_{\pi} \hat{w}_{\pi}\left(s_{\partial \Omega(\pi)}\left(\vec{x}_{\gamma_{\pi}}, \vec{u}_{\gamma_{\pi}}\right)\right)
$$

At this point the boundary conditions of the sensitivity model are unknown. The main point of constructing the sensitivity model is to formulate the boundary conditions. In [7], it is demonstrated that the boundary condition of the sensitivity model is a linear application $\mathcal{L}$ of the radiative intensity and the

\footnotetext{
${ }^{1}$ Formulating $J(\pi)$ as a linear integral function of radiative intensity $J(\pi)=f(I(\vec{x}, \vec{u}, \pi))$ leads to $J(\pi)=$ $f\left[\mathbb{E}\left[\hat{w}_{J}\left(I_{\partial \Omega(\pi)}\left(\vec{x}_{\Gamma}, \vec{u}_{\Gamma}\right)\right)\right]\right](7)$. Now when derivating $J(\pi)$ we obtain $\partial_{\pi} J(\pi)=g\left(\partial_{\pi} I(\vec{x}, \vec{u}, \pi)\right)$ a linear integral function of the radiative intensity derivative.
} 
sensitivity transmitted at the boundary.

$$
s_{\partial \Omega(\pi)}\left(\vec{x}_{\gamma_{\pi}}, \vec{u}_{\gamma_{\pi}}, \pi\right)=\mathcal{L}\left[I\left(\vec{x}_{\gamma_{\pi}}, \vec{u}^{\prime}, \pi\right), s\left(\vec{x}_{\gamma_{\pi}}, \vec{u}_{\gamma_{\pi}}^{\prime}, \pi\right)\right]
$$

Dauchet[7] provides formulations of the linear application $\mathcal{L}$ for two generic sensitivity boundary condi-

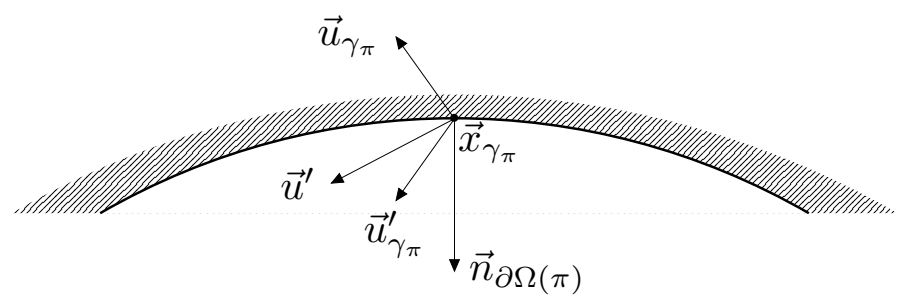

Figure 1. Schema corresponding to the sensitivity boundary condition (Eq. (10))

tions in consideration of two different radiative boundary conditions: absorbing, non emitting boundary conditions; emitting and reflective boundary conditions. In each case sensitivities are estimated in academic configurations.

\section{IMPLEMENTATION}

To illustrate the previous proposal, let us consider a square cavity containing a gray semi-transparent medium. Square dimensions are set along the $x$ and $y$ axes and are infinite along the $z$ axis. At the center of the square an infinite black-body cylinder emits radiation (Figure 2). The model domain definition is stated as $\Omega$, and its boundary $\partial \Omega(\pi)$ is constituted by a cavity boundary $\mathcal{R}(\pi)$ and a cylinder black body boundary $\mathcal{F} . \vec{n}$ is a normal vector at the boundary $\partial \Omega(\pi)$ and oriented towards the medium $\Omega$. $\pi$ is a geometrical parameter defining the cavity boundary $\mathcal{R}(\pi)$. In this example we consider a colinear trans-
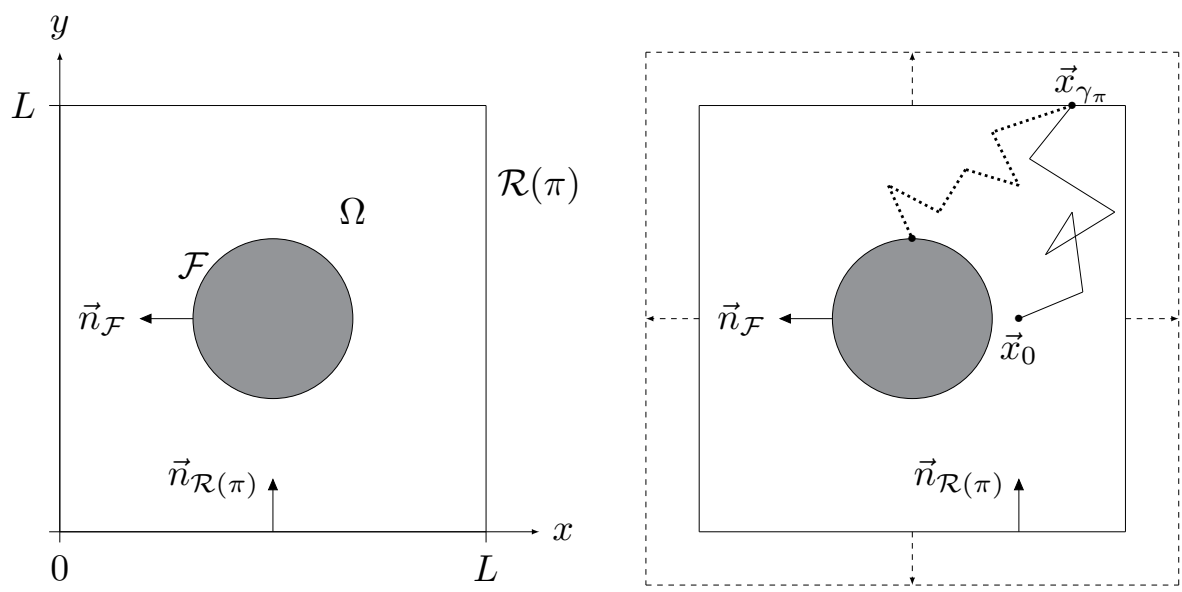

Figure 2. On the left, the square cavity geometry filled by a semi-transparent medium, lightened by an emissive cylinder at its center. On the right, the dashed line represent the scaling of the cavity and the dotted line the coupling between the sensitivity boundary condition and radiative intensity.

lation transformation of $\mathcal{R}(\pi)$ according to normal vector $\vec{n}_{\mathcal{R}(\pi)}$ resulting in a scaling of the cavity. The cavity and cylinder radiative boundary properties are black body. The cavity is non-emitting while the cylinder is emitting. In this example, absorbed radiative heat transfer density $J\left(\vec{x}_{0}, \pi\right)$ and its derivative $\partial_{\pi} J\left(\vec{x}_{0}, \pi\right)$ are estimated.

$$
\begin{gathered}
J\left(\vec{x}_{0}, \pi\right)=\int_{4 \pi} k_{a} I\left(\vec{x}_{0}, \vec{u}_{0}, \pi\right) d \overrightarrow{u_{0}} \\
\partial_{\pi} J\left(\vec{x}_{0}, \pi\right)=\int_{4 \pi} k_{a} s\left(\vec{x}_{0}, \vec{u}_{0}, \pi\right) d \overrightarrow{u_{0}}
\end{gathered}
$$


In this configuration, the radiative model is constituted by the RTE (5) in the stationary state and its boundary conditions :

$$
\left\{\begin{array}{l}
I\left(\vec{x}_{\mathcal{R}}, \vec{u}, \pi\right)=0 \\
I\left(\vec{x}_{\mathcal{F}}, \vec{u}\right)=I_{b}(T)
\end{array}\right.
$$

Absorbed radiative heat transfer density $J\left(\vec{x}_{0}, \pi\right)$ is an integral function of radiative intensity at location $\vec{x}_{0}$ and $I\left(\vec{x}_{0}, \vec{u}_{0}, \pi\right)$ is the integral radiative transfer equation solution at location $\vec{x}_{0}$ and direction $\vec{u}_{0}$.

$$
J\left(\vec{x}_{0}, \pi\right)=\int_{4 \pi} d w_{0} \int_{\mathcal{D}_{\Gamma}} d \gamma p_{\Gamma}\left(\vec{x}_{\gamma}, \vec{u}_{\gamma} \mid \vec{x}_{0}, \vec{u}_{0}\right) \hat{w}\left(I_{\partial \Omega(\pi)}\left(\vec{x}_{\gamma}, \vec{u}_{\gamma}\right)\right)
$$

In equation (14) $\Gamma$ represents a random optical path in the scattering medium until it reaches a location and direction $\left(\vec{x}_{\gamma}, \vec{u}_{\gamma}\right)$ at the boundary (Figure 2 ). With regard to the configuration transformation and considering the boundary radiative properties, the linear application $\mathcal{L}$ describing sensitivity boundary condition is [7]:

$$
s_{\partial \Omega(\pi)}\left(\vec{x}_{\mathcal{R}(\pi)}, \vec{u}, \pi\right)=-\frac{k_{s}}{\vec{u} \cdot \vec{n}_{\mathcal{R}}} \int_{2 \pi^{-}} p\left(\vec{u} \mid \vec{u}^{\prime}\right) d \vec{u}^{\prime} I\left(\vec{x}, \vec{u}^{\prime}, \pi\right)
$$

Equation (15) is stated on the $\mathcal{R}(\pi)$ boundary and for the $\mathcal{F}$ boundary:

$$
s_{\partial \Omega(\pi)}\left(\vec{x}_{\mathcal{F}}, \vec{u}\right)=0
$$

Since $s\left(\vec{x}_{0}, \vec{u}_{0}, \pi\right)$ is the solution to the integral sensitivity transport equation it can be stated, both as the radiative intensity and as the expectation of the Monte-Carlo weight function $\hat{w}_{\pi}$ depending on the sensitivity boundary conditions (17).

$$
\partial_{\pi} J\left(\vec{x}_{0}, \pi\right)=\int_{4 \pi} p_{\vec{U}}\left(\vec{u}_{0}\right) d u_{0} \int_{\mathcal{D}_{\Gamma_{\pi}}} d \gamma_{\pi} p_{\Gamma_{\pi}}\left(\vec{x}_{\gamma_{\pi}}, \vec{u}_{\gamma_{\pi}} \mid \vec{x}_{0}, \vec{u}_{0}\right) \hat{w}_{\pi}\left(s_{\partial \Omega(\pi)}\left(\vec{x}_{\gamma_{\pi}}, \vec{u}_{\gamma_{\pi}}\right)\right)
$$

The purpose of the following part is to outline the algorithm details that come with the formulation of equation (17). Figure 2 illustrates the transport of sensitivity coupled with the radiative intensity at the boundary. This coupling comes from the dependence of $s_{\partial \Omega(\pi)}$ to $I(\vec{x}, \vec{u}, \pi)$ (as shown in Eq. 13). The quantity to evaluate is the absorbed radiative heat transfer density sensitivity $\partial_{\pi} J\left(\vec{x}_{0}, \pi\right)$. The transport phenomenon algorithm used in this work is based on the path-length method [14]. The path length are generated under the assumption of pure scattering and the quantity is then attenuated over the scattering path length [14].

The process of sampling the random variable $\Gamma_{\pi}$ from the probability density function $p_{\Gamma_{\pi}}\left(\vec{x}_{\gamma_{\pi}}, \vec{u}_{\gamma_{\pi}} \mid \vec{x}_{0}, \vec{u}_{0}\right)$ consists on the realization of a sensitivity path, that is a realization $\gamma_{\pi}$ of $\Gamma_{\pi}$. This implies sampling sequentially, for the $(n+1)$ th scattering event, a direction from the phase probability density function $p_{\vec{U}_{n+1}}\left(\vec{u}_{n+1} \mid \vec{u}_{n}\right)$ and a position from the exponential Beer-Lambert law probability density function $p_{L_{n+1}}\left(\vec{x}_{n+1}=\vec{x}_{n}-l_{n+1} \vec{u}_{n+1}, \vec{u}_{n+1} \mid \vec{x}_{n}, \vec{u}_{n}\right)[1,8]$. This process continues until the sensitivity path reaches the boundary at location $\vec{x}_{\gamma_{\pi}}$ with direction $\vec{u}_{\gamma_{\pi}}$.

At that point, if the boundary is dependent on the geometrical parameter $\pi$, the sensitivity boundary condition (15) is coupled with the radiative intensity at location $\vec{x}_{\gamma_{\pi}}$ and direction $\vec{u}^{\prime}$. A direction $\vec{u}^{\prime}$ is sampled from the isotropic probability density function at the boundary. If the direction is oriented outside of the cavity domain (scalar product $\vec{u}^{\prime} \cdot \vec{n}_{\mathcal{R}(\pi)}<0$ ), the radiative intensity value is the radiative boundary condition at $\mathcal{R}(\pi)$ (box B in Figure 3). Otherwise a radiative path $\gamma$ of random variable $\Gamma$ is sampled until it reaches the boundary again (box $\mathrm{C}$ and D in Figure 3). In this example, the sensitivity path (continuous line) and the radiative intensity path (dashed line) drawn in Figure 2 are both describing the only configuration where a realization of sensitivity Monte-Carlo weight function would have a non null value (box $\mathrm{C}$ in Figure 3). 


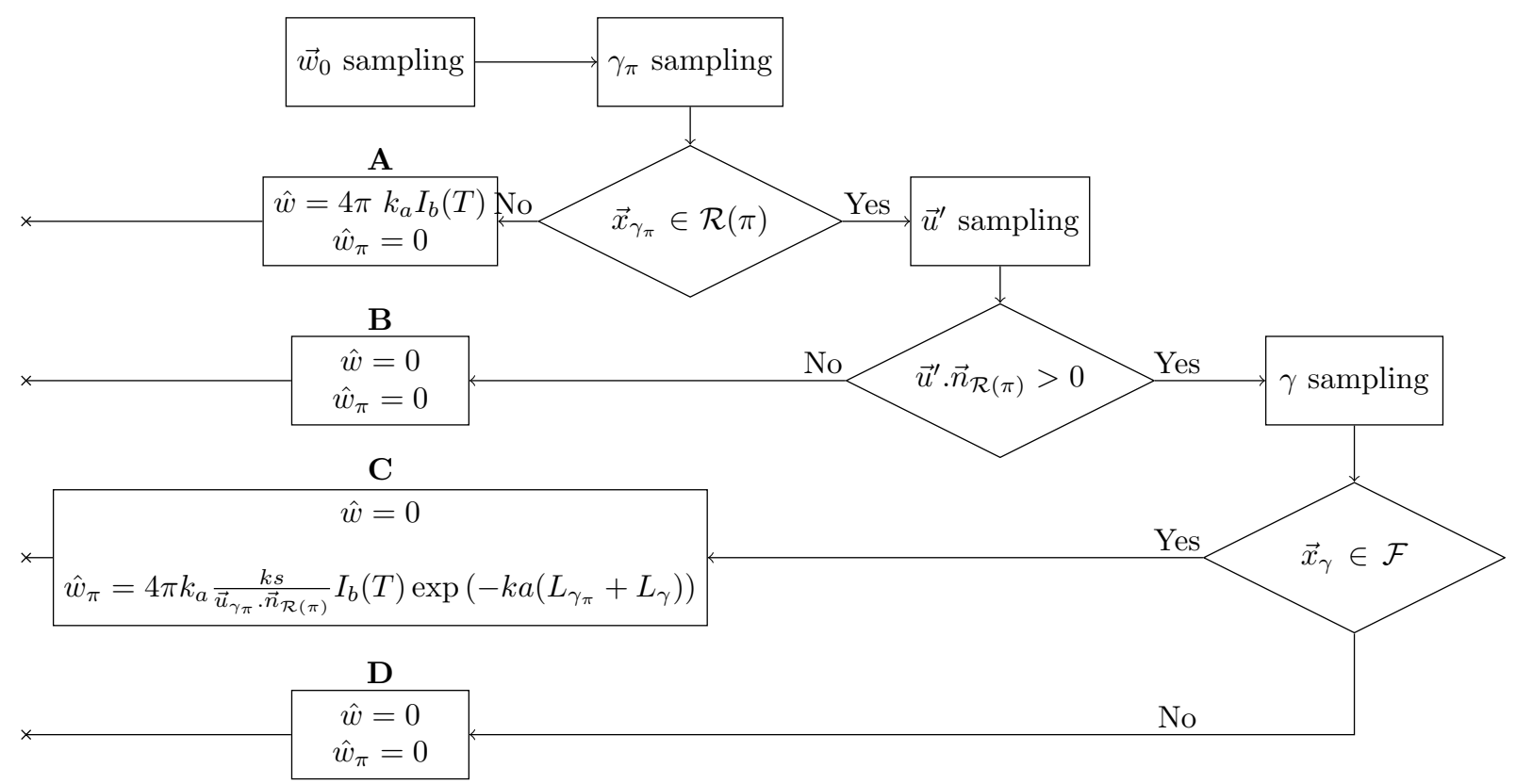

Figure 3. Algorithmic translation of equation (17)

\section{RESULTS}

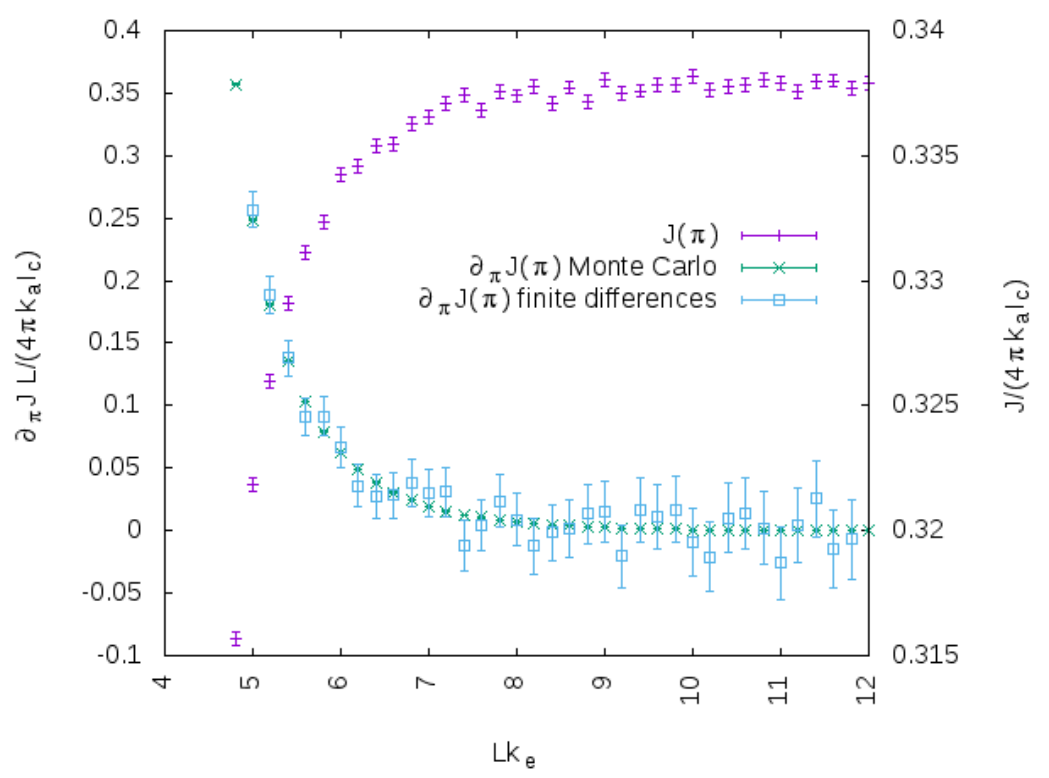

Figure 4. Absorbed radiative intensity density $J(\pi)$ and its sensitivity $\partial_{\pi} J(\pi)$ to the normal transformation of the $\mathcal{R}(\pi)$ boundary. The phase function in a semi-transparent medium is isotropic $p_{\vec{U}}\left(\vec{u}_{i}\right)=\frac{1}{4 \pi}$ and the probability density function of scattering extinction is $p_{\Sigma}\left(l_{i}\right)=k_{s} \exp \left(-k_{s} l_{i}\right)$. Cavity dimensions are set by a variation $\delta L$ of $L$ influencing the optical thickness $k_{e} L$. Single scattering albedo is uniform on the cavity $\frac{k_{s}}{k_{e}}=0.5$. Estimations of the absorbed radiative intensity density and its sensitivity are obtained for $2.10^{6}$ realizations $N$ of the corresponding Monte-Carlo weight function.

Figure 4 displays the results of the algorithm presented in Figure 3. In order to validate the sensitivity model method, the finite differences are calculated from Monte-Carlo estimations of absorbed radiative intensity density. Figure 4 shows the difficulty to evaluate the sensitivities from finite differences. If $\delta L$ value is chosen small in regards to $L$, the variance of finite difference becomes too large and the results does not converge. On the contrary if $\delta L$ is chosen too large in regards to $L$, the calculation of the finite difference converges but the value of the sensitivity is not relevant. This difficulty is well known when gradients are estimated by differentiation, it is outlined in the gradient-based Kiefer-Wolfowitz method 
Table 1: Absorbed radiative intensity density and its sensitivity results for a fixed value of $L$. The probability density function for isotropic scattering is $p_{\vec{U}}\left(\vec{u}_{i}\right)=\frac{1}{4 \pi}$ and probability density function of scattering extinction is $p_{\Sigma}\left(l_{i}\right)=k_{s} \exp \left(-k_{s} l_{i}\right)$. Location $\vec{x}_{0}$ on the $y$ axis is determined by $\frac{y_{0}}{L}=0.5$ and the ratio between the cylinder radius and $\mathrm{L}$ is set at $\frac{r}{L}=0.125$. The number of realizations of the Monte-Carlo weight functions is $N=2 \times 10^{6}, \epsilon(\%)$ represents the relative error of $\partial_{\pi} J(\pi)$ and $J(\pi)$.

\begin{tabular}{|ccccccccc|}
\hline $\boldsymbol{k}_{\boldsymbol{a}} \boldsymbol{L}$ & $\boldsymbol{k}_{\boldsymbol{s}} \boldsymbol{L}$ & $\frac{\boldsymbol{x}_{\mathbf{0}}}{\boldsymbol{L}}$ & $\frac{\boldsymbol{\partial}_{\pi} \boldsymbol{J}\left(\overrightarrow{\boldsymbol{x}}_{0}, \boldsymbol{\pi}\right) \boldsymbol{L}}{4 \pi \boldsymbol{k}_{a} \boldsymbol{I}_{\boldsymbol{b}} \boldsymbol{m a x}}$ & $\frac{\boldsymbol{\sigma} \boldsymbol{L}}{4 \pi \boldsymbol{k}_{a} \boldsymbol{I}_{b} \boldsymbol{m a x}}$ & $\epsilon_{\partial_{\pi} J}(\%)$ & $\frac{\boldsymbol{J}_{\left(\overrightarrow{\boldsymbol{x}}_{0}, \boldsymbol{\pi}\right)}^{4 \pi \boldsymbol{k}_{a} \boldsymbol{I} \boldsymbol{m a x}}}{}$ & $\frac{\sigma}{4 \pi \boldsymbol{k}_{a} \boldsymbol{I}_{b} \boldsymbol{m a x}}$ & $\epsilon_{J}(\%)$ \\
\hline 1 & 1 & 0.6375 & $1.47 \mathrm{e}-02$ & $6.221 \mathrm{e}-05$ & 0.42 & $3.89 \mathrm{e}-01$ & $3.297 \mathrm{e}-04$ & 0.09 \\
1 & 10 & 0.6375 & $1.88 \mathrm{e}-02$ & $2.299 \mathrm{e}-04$ & 0.01 & $5.54 \mathrm{e}-01$ & $3.037 \mathrm{e}-04$ & 0.06 \\
1 & 50 & 0.6375 & $4.06 \mathrm{e}-04$ & $2.765 \mathrm{e}-05$ & 6.80 & $6.64 \mathrm{e}-01$ & $2.665 \mathrm{e}-04$ & 0.04 \\
1 & 100 & 0.6375 & $1.08 \mathrm{e}-05$ & $2.31 \mathrm{e}-06$ & 21.31 & $6.66 \mathrm{e}-01$ & $2.602 \mathrm{e}-04$ & 0.04 \\
1 & 0.001 & 0.6375 & $1.85 \mathrm{e}-05$ & $6.243 \mathrm{e}-08$ & 0.34 & $3.53 \mathrm{e}-01$ & $3.308 \mathrm{e}-04$ & 0.09 \\
10 & 1 & 0.6375 & $1.34 \mathrm{e}-06$ & $1.198 \mathrm{e}-08$ & 0.89 & $2.86 \mathrm{e}-01$ & $2.669 \mathrm{e}-04$ & 0.09 \\
50 & 1 & 0.6375 & $1.16 \mathrm{e}-20$ & $3.874 \mathrm{e}-22$ & 3.33 & $1.19 \mathrm{e}-01$ & $1.292 \mathrm{e}-04$ & 0.01 \\
0.001 & 1 & 0.6375 & $6.50 \mathrm{e}-02$ & $2.878 \mathrm{e}-04$ & 0.44 & $4.23 \mathrm{e}-01$ & $3.493 \mathrm{e}-04$ & 0.08 \\
1 & 1 & 0.75 & $2.24 \mathrm{e}-02$ & $9.048 \mathrm{e}-05$ & 0.41 & $1.54 \mathrm{e}-01$ & $2.199 \mathrm{e}-04$ & 0.14 \\
1 & 1 & 0.875 & $3.27 \mathrm{e}-02$ & $1.255 \mathrm{e}-04$ & 0.38 & $8.10 \mathrm{e}-02$ & $1.531 \mathrm{e}-04$ & 0.19 \\
1 & 1 & 0.9875 & $7.70 \mathrm{e}-02$ & $3.644 \mathrm{e}-04$ & 0.47 & $4.73 \mathrm{e}-02$ & $1.123 \mathrm{e}-04$ & 0.24 \\
1 & 1 & $0.9875=\frac{y_{0}}{L}$ & $5.22 \mathrm{e}-02$ & $3.287 \mathrm{e}-04$ & 0.63 & $2.05 \mathrm{e}-02$ & $6.766 \mathrm{e}-05$ & 0.33 \\
\hline
\end{tabular}

presented in [4]. The sensitivity model has been also cross-validated with deformation velocity method on academical configurations. It also shows that the absorbed radiative intensity density becomes less sensitive to a variation of $\frac{\delta L}{L}$ when the size of the cavity increases. Table 1 summarizes the results of absorbed radiative intensity density and its shape sensitivity for a different set of parameters. Table 1 shows that the closer the probe point gets to the $\mathcal{R}(\pi)$ boundary the higher the sensitivity is. It also shows a limit to the evaluation of the sensitivities in the case of an important optical thickness, in the other cases there are no convergence issues.

\section{CONCLUSION}

When considering Monte-Carlo sensitivities, the derivatives of an integral quantity with respect to a geometrical parameter lead to different solution methods. In this work, we present a new approach to geometrical sensitivity estimation. This methodology allows one to consider problems that previously have been technically unsolvable by the deformation velocity method. However, this development comes with a cost in terms of the algorithm. While the deformation velocity method made it possible to compute the objective function and its sensitivity at the same time, the sensitivity model method leads to a different and more complex algorithm for the objective function sensitivity than the objective function itself. But since both sensitivity and intensity share the same transport model, the objective function and its sensitivity are usually estimated simultaneously. Nevertheless, an additional simulation time is still required for the radiative intensity coupling at the sensitivity boundary condition.

For now, the sensitivity model has been implemented only for a local quantity, because of the integration domain dependence on the geometrical parameter in the formulation of the global quantity integral. This particularity generates a new kind of algorithm to explore with the sensitivity model method. Other technical problems are under study. For instance, the linear application of the sensitivity boundary condition is also stated for specular reflective conditions, but it involves knowing the radiative intensity gradient at the boundary [7].

So far we have focused on one type of shape transformation, which is the normal vector colinear translation. Although this work is still in progress, the algorithm presented in Figure 3 has already been implemented on several complex geometry applications such as solar heat flux sensitivity with respect 
to heliostat size in a concentrated solar tower [3]. The academic example presented in this work is based on a simplified porous medium geometry that can be applied on photobioreactors [7] and in a hightemperature solar volumetric receiver [15].

\section{ACKNOWLEDGMENTS}

This project has received funding from the Occitanie region and University of Perpignan Via Domitia, from the programme investments for the future of the French National Agency for Research (ANR) under award number ANR-10-LBX-22-01-SOLSTICE. We acknowledge support from the ANR, grant HIGH-TUNE ANR-16-CE01-0010, (http://www.umr- cnrm.fr/high-tune and ANR, grant MGC-RAD) and from Region Occitanie (Projet CLE-2016 ED- Star).

\section{REFERENCES}

[1] J. Delatorre, G. Baud, J.-J. Bézian, S. Blanco, C. Caliot, J.-F. Cornet, C. Coustet, J. Dauchet, M. El Hafi, V. Eymet et al., "Monte carlo advances and concentrated solar applications," Solar Energy, vol. 103, pp. 653-681, 2014.

[2] S. A. Rukolaine, "The shape gradient of the least-squares objective functional in optimal shape design problems of radiative heat transfer," Journal of Quantitative Spectroscopy and Radiative Transfer, vol. 111, no. 16, pp. 2390-2404, 2010.

[3] O. Farges, "Conception optimale de centrales solaires à concentration: application aux centrales à tour et aux installations" beam down"," Ph.D. dissertation, Ecole nationale des Mines d'Albi-Carmaux, 2014.

[4] K. Daun, D. Morton, and J. Howell, "Geometric optimization of radiant enclosures containing specular surfaces," Journal of Heat Transfer, vol. 125, no. 5, pp. 845-851, 2003.

[5] J. De la Torre, "Calculs de sensibilités par méthode de monte-carlo, pour la conception de procédés à énergie solaire concentrée," Ph.D. dissertation, INPT, 2011.

[6] N. Villefranque, F. Couvreux, R. Fournier, S. Blanco, C. Cornet, V. Eymet, V. Forest, and J.-M. Tregan, "Path-tracing monte carlo libraries for $3 \mathrm{~d}$ radiative transfer in cloudy atmospheres," submitted to Journal of Advances in Modeling Earth Systems, 2019.

[7] J. Dauchet, "Analyse radiative des photobioréacteurs," Ph.D. dissertation, Université Blaise Pascal-Clermont-Ferrand II, 2012.

[8] J. Dauchet, S. Blanco, J.-F. Cornet, M. E. Hafi, V. Eymet, and R. Fournier, "The practice of recent radiative transfer monte carlo advances and its contribution to the field of microorganisms cultivation in photobioreactors," Journal of Quantitative Spectroscopy and Radiative Transfer, vol. 128, pp. 52 - 59, 2013, eurotherm Seminar on Computational Thermal Radiation in Participating Media IV. [Online]. Available: http://www.sciencedirect.com/science/article/pii/S0022407312003299

[9] W. L. Dunn and J. K. Shultis, Exploring monte carlo methods. Elsevier, 2011.

[10] A. de Lataillade, S. Blanco, Y. Clergent, J. Dufresne, M. E. Hafi, and R. Fournier, "Monte carlo method and sensitivity estimations," Journal of Quantitative Spectroscopy and Radiative Transfer, vol. 75, no. 5, pp. 529 - 538, 2002. [Online]. Available: http://www.sciencedirect.com/science/article/pii/S0022407302000274

[11] M. Roger, M. El Hafi, R. Fournier, S. Blanco, A. de Lataillade, V. Eymet, and P. Perez, "Application of sensitivity estimations by monte carlo methods," in Proceeding of the 4th International Symposium on Radiative Transfer. Begellhouse, 2004, pp. 1-8.

[12] M. Roger, S. Blanco, M. El Hafi, and R. Fournier, "Monte carlo estimates of domain-deformation sensitivities," Phys. Rev. Lett., vol. 95, p. 180601, Oct 2005. [Online]. Available: https://link.aps.org/doi/10.1103/PhysRevLett.95.180601

[13] M. Roger, "Modèles de sensibilité dans le cadre de la méthode de monte-carlo : illustrations en transfert radiatif," Ph.D. dissertation, INPT, 2006.

[14] V. Eymet, D. Poitou, M. Galtier, M. E. Hafi, G. Terre, and R. Fournier, "Null-collision meshless montecarloapplication to the validation of fast radiative transfer solvers embedded in combustion simulators," Journal of Quantitative Spectroscopy and Radiative Transfer, vol. 129, pp. 145 - 157, 2013. [Online]. Available: http://www.sciencedirect.com/science/article/pii/S0022407313002483

[15] S. Mey-Cloutier, C. Caliot, A. Kribus, Y. Gray, and G. Flamant, "Experimental study of ceramic foams used as high temperature volumetric solar absorber," Solar Energy, vol. 136, pp. 226 - 235, 2016. [Online]. Available: http://www.sciencedirect.com/science/article/pii/S0038092X1630247X 